\title{
Continuous invariant cone families and ergodicity of flows in dimension three
}

\author{
KEITH BURNS AND MARLIES GERBER \\ Department of Mathematics, Indiana University, Bloomington, IN 47405, USA
}

(Received 12 June 1987 and revised 29 June 1987)

\begin{abstract}
It is shown that a $C^{2}$ flow on a compact three-dimensional manifold that preserves a smooth measure and has a continuous family of cones satisfying a certain invariance property must be ergodic.
\end{abstract}

\section{Introduction}

By the results of $M$. Wojtkowski [W], any flow on a compact Riemannian manifold $M$ which preserves a smooth measure and has a measurable family of cones satisfying a certain invariance condition must have at least one nonzero Lyapunov exponent almost everywhere. In the case when $M$ is three dimensional this implies that all Lyapunov exponents (except the one corresponding to the flow direction) are nonzero almost everywhere. It then follows from the theory of Pesin $[\mathbf{P}]$ that the flow has an ergodic component of positive measure. Here we prove that if $M$ is three dimensional and we have a continuous family of cones on a connected open subset $U$ of $M$ such that Wojtkowski's invariance condition is satisfied for the flow induced on $U$, and the orbit of almost every point passes through $U$, then the flow is ergodic. We show that the stable and unstable foliations guaranteed by $[P]$ fill up (modulo a subset of measure zero) with 'long' leaves some neighborhood of every point in $U$. The continuity of the family of cones is used to force uniform transversality of stable and unstable manifolds in such neighborhoods.

The main new application of our result is to prove the existence of real analytic ergodic geodesic flows on $S^{2}$ [BG2]. V. Donnay [D1], [D2], [D3] had already found $C^{\infty}$ geodesic flows on $S^{2}$ with nonzero Lyapunov exponents almost everywhere, and he also has written a different proof of ergodicity in the $C^{\infty}$ case [D4], based on an outline in [D2]. His technique seems to involve a direct construction of stable and unstable manifolds for his particular examples.

A. Katok [K] has recently extended our method to higher dimensions. He showed that under similar assumptions on a continuous cone family, symplectic diffeomorphisms and flows preserving a contact form are ergodic and, in fact, Bernoulli. The results of [W] and [P] also apply in this situation. Katok's proof uses a new 'noncontraction' theorem for unstable manifolds that was not needed for our case, in which the unstable manifolds are one dimensional.

An earlier version of the result in the present paper was circulated in [BG1]. 
We thank M. Brin and A. Katok for helpful conversations.

The second author is grateful to the Sonderforschungsbereich, Göttingen for support and warm hospitality during the time this paper was written.

\section{Formulation of the result}

Let $M$ be a $C^{3}$, compact, connected, three dimensional Riemannian manifold and $\mu$ a measure on $M$ that is equivalent to the Riemannian volume. Consider a $C^{2}$ flow $\varphi^{t}$ on $M$ that leaves $\mu$ invariant. Let $X$ be the vector field that generates $\varphi^{\prime}$.

A cone $\mathscr{K}$ in a real two dimensional vector space is a subset $\mathscr{K}=\mathscr{K}(v, w)=$ $\{a v+b w: a b \geq 0\}$ where $v$ and $w$ are linearly independent vectors and $a, b \in \mathbb{R}$. We call Int $(\mathscr{K})=\{a v+b w: a b>0$ or $a=b=0\}$ the interior of $\mathscr{K}$ and $\mathscr{K}^{*}=$ $\{a v+b w: a b \leq 0\}$ the complement of $\mathscr{K}$. If $P$ is a two dimensional distribution defined on a subset $S$ of $M$, a family of cones in $P$ over $S$ is a set $\{\mathscr{K}(x): x \in S\}$ such that $\mathscr{K}(x)$ is a cone in $P(x)$ for each $x \in S$. The family is continuous if $S$ is covered by relatively open subsets $S_{i}$ on each of which there are continuous vector fields $v_{i}$ and $w_{i}$ with $\mathscr{K}(x)=\mathscr{K}\left(v_{i}(x), w_{i}(x)\right)$ for each $x \in S_{i}$.

THEOREM 1.1. Let $U \subseteq M$ be a connected open set with $X(x) \neq 0$ for every $x \in U$. Suppose there is a continuous two dimensional distribution $P$ defined on $U$ and $a$ continuous family $\mathscr{K}(x)$ of cones in $P$ over $U$ such that

(i) $P(x)$ and $X(x)$ span $T_{x} M$ for each $x \in U$;

(ii) $d \varphi^{t} P(x)=P\left(\varphi^{t} x\right)$ whenever $x \in U$ and $\varphi^{t} x \in U$;

(iii) $d \varphi^{t} \mathscr{K}(x) \subseteq \mathscr{K}\left(\varphi^{t} x\right)$ whenever $x \in U, \varphi^{t} x \in U$ and $t \geq 0$;

(iv) for each $x \in U$ there is $t(x)>0$ such that $\varphi^{t(x)} x \in U$ and $d \varphi^{t(x)} \mathscr{K}(x) \subseteq$ Int $\left(\mathscr{K}\left(\varphi^{t(x)} x\right)\right)$.

Then the flow $\varphi^{\prime}$ is ergodic with respect to $\mu$ on $\hat{U}=\bigcup\left\{\varphi^{\prime} U: t \in \mathbb{R}\right\}$.

Remarks. (i) If the orbit of $\mu$-a.e. point of $M$ enters $U$, then $\varphi^{\prime}$ is ergodic on $M$.

(ii) An analogous theorem holds for diffeomorphisms of surfaces. The proof of Theorem 1.1 carries over easily to this case.

The remainder of this paper is devoted to the proof of Theorem 1.1. In $\S 2$ we summarize and reformulate the results from $[P]$ and $[W]$ that we need. In $\S 3$ we complete the proof of Theorem 1.1.

\section{Stable and unstable manifolds}

In the rest of this paper we shall assume the hypothesis of Theorem 1.1.

Osceledec's multiplicative ergodic theorem [O] implies that for $\mu$-a.e. $x \in M$ the Lyapunov exponents of $v$

$$
\chi^{+}(v)=\lim _{t \rightarrow+\infty} \frac{1}{t} \log \left\|d \varphi^{\prime} v\right\| \quad \text { and } \quad \chi^{-}(v)=\lim _{t \rightarrow-\infty} \frac{1}{t} \log \left\|d \varphi^{\prime} v\right\|
$$

are defined for every nonzero $v \in T_{x} M$. We then have the following result, which is essentially contained in [W].

THeOrem 2.1. For $\mu$-a.e. $x \in U$, we have $\chi^{+}(v)>0$ for every $v \in \operatorname{Int}(K(x))$ and $\chi^{-}(v)<0$ for every $v \in \operatorname{Int}\left(K^{*}(x)\right)$. 
Proof. This follows from $\S 2$ of [W], although the results there are not formally stated for the fiow case. To pass from the discrete time case to the flow case here, simply note that Wojtkowski's conditions are satisfied by the first return map $\left(\varphi^{1}\right)_{U}$ of the time-one map $\varphi^{1}$ to $U$.

THEOREM 2.2. There is a $\varphi^{t}$-invariant set $\Lambda \subseteq \hat{U}$ with $\mu(\hat{U} \backslash \Lambda)=0$ on which there is a $d \varphi^{t}$-invariant splitting of $T_{x} M$ into one dimensional subbundles, $T_{x} M=E^{0}(x) \oplus$ $E^{s}(x) \oplus E^{u}(x), x \in \Lambda$, where for each $x \in \Lambda$

(i) $E^{0}(x)$ is spanned by $X(x)$;

(ii) $\chi^{+}(v)=\chi^{-}(v)<0$ if $v \in E^{s}(x) \backslash\{0\}$;

(iii) $\chi^{+}(v)=\chi^{-}(v)>0$ if $v \in E^{u}(x) \backslash\{0\}$;

(iv) $E^{u}(x) \subseteq \mathscr{K}(x)$ and $E^{s}(x) \subseteq \mathscr{K}^{*}(x)$ if $x \in \Lambda \cap U$.

Proof. Properties (i), (ii), (iii) are Osceledec's theorem [O] and property (iv) follows from properties (ii), (iii) and Theorem 2.1.

If $x \in M$, the global stable manifold of $x$ is the set

$$
W^{s}(x)=\left\{y \in M: \operatorname{dist}\left(\varphi^{\prime} y, \varphi^{t} x\right) \rightarrow 0 \text { exponentially as } t \rightarrow+\infty\right\}
$$

and the global unstable manifold is

$$
W^{u}(x)=\left\{y \in M: \operatorname{dist}\left(\varphi^{-t} y, \varphi^{-t} x\right) \rightarrow 0 \text { exponentially as } t \rightarrow+\infty\right\} .
$$

If $x \in M$, define its weak stable manifold

$$
W^{0 s}(x)=\bigcup\left\{\varphi^{t} W^{s}(x): t \in \mathbb{R}\right\} .
$$

For $\mu$-a.e. $x \in \Lambda, W^{s}(x)$ and $W^{u}(x)$ are $C^{1}$-immersed submanifolds that are tangent to the distributions $E^{s}$ and $E^{u}$ respectively. More precisely, the following result holds:

TheOrem 2.3. (Pesin) There is a $\varphi^{t}$-invariant subset $\tilde{\Lambda}$ of $\Lambda$ with $\mu(\Lambda \backslash \tilde{\Lambda})=0, a$ measurable function $\delta: \tilde{\Lambda} \rightarrow \mathbb{R}^{+}$and measurable maps

$$
\alpha^{s}, \alpha^{u}:\{(x, r) \in \tilde{\Lambda} \times \mathbb{R}:|r| \leq \delta(x)\} \rightarrow M
$$

such that, for each $x \in \tilde{\Lambda}, \alpha^{s}(x, 0)=x=\alpha^{u}(x, 0)$ and $\alpha^{s}(x, \cdot), \alpha^{u}(x, \cdot)$ are $C^{1}$ isometric embeddings of $[-\delta(x), \delta(x)]$ into $M$, whose images $V^{s}(x)$ and $V^{u}(x)$ respectively satisfy the following properties for all $x \in \tilde{\Lambda}$ :

(i) $V^{u}(x) \subseteq W^{u}(x)$;

(ii) $W^{u}(x)=\bigcup\left\{\varphi^{t} V^{u}\left(\varphi^{-t} x\right): t \geq 0\right\}$;

(iii) $T_{x} V^{u}(x)=E^{u}(x)$;

(iv) $\varphi^{t} V^{u}(x) \subseteq W^{u}\left(\varphi^{t} x\right)$ for any $t$;

(v) if $y \in V^{u}(x) \cap \tilde{\Lambda}$ and $y=\alpha^{u}\left(x, r_{0}\right)$, then $\alpha^{u}\left(y, r-r_{0}\right)=\alpha^{u}(x, r)$ whenever both sides are defined;

(vi) if $y \in W^{u}(x)$ and $\beta$ is the piece of $W^{u}(x)$ joining $x$ to $y$ then the length of $\varphi^{-t} \beta$ approaches zero as $t \rightarrow \infty$;

(vii) the analogues of (i)-(vi) hold for $\alpha^{s}, V^{s}$ and $W^{s}$.

Proof. This follows from the analogues for flows of Theorems 4.1, 4.2 and 6.1 of [P] and Theorem 17 of [FHY]. These flow analogues are discussed in $\$ 9$ of $[P]$. 
We shall call $V^{s}(x)$ and $V^{u}(x)$ the local stable and unstable manifolds of $x$, respectively. Their behavior can be uniformly controlled outside subsets of arbitrarily small measure. More precisely, we have:

THEOREM 2.4. (Pesin) For each $\varepsilon>0$ there is a closed subset $\tilde{\Lambda}_{\varepsilon} \subseteq \tilde{\Lambda}$ such that

(i) $\delta(x) \geq \varepsilon$ for all $x \in \tilde{\Lambda}_{\varepsilon}$;

(ii) $\delta$ is continuous on $\tilde{\Lambda}_{\varepsilon}$;

(iii) $V^{s}(x)$ and $V^{u}(x)$ vary continuously in the $C^{1}$ topology for $x \in \tilde{\Lambda}_{\varepsilon}$;

(iv) if $x \in \tilde{\Lambda}_{\varepsilon}, v \in E^{s}(x), w \in E^{u}(x)$ and $t \geq 0,\left\|d \varphi^{-t} v\right\| \geq \varepsilon(1+\varepsilon)^{t}$ and $\left\|d \varphi^{t} w\right\| \geq$ $\varepsilon(1+\varepsilon)^{t}$

(v) $\mu\left(\tilde{\Lambda} \backslash \tilde{\Lambda}_{\varepsilon}\right) \rightarrow 0$ as $\varepsilon \rightarrow 0$.

Proof. This follows from the analogues for flows of Proposition 4.1 and Theorems 4.2 and 4.3 in $[\mathbf{P}]$. See $\$ 9$ of [P].

The 'foliations' of local stable and unstable manifolds have the property of absolute continuity. We formulate this only for $V^{u}$, since the situation for $V^{s}$ is analogous. Let $x \in \tilde{\Lambda}_{\varepsilon}$ be a density point of $\tilde{\Lambda}_{\varepsilon}$, and $B$ a ball with center $x$. Define a $\tilde{\Lambda}_{\varepsilon}$-transversal to $V^{\prime \prime}(x)$ in $B$ to be a $C^{1}$ submanifold $T$ of $B$ such that

(i) $T$ intersects $V^{u}(x)$.

(ii) $T \cap V^{u}(y)$ contains at most one point for each $y \in B \cap \tilde{\Lambda}_{\varepsilon}$.

(iii) Any intersection of $T$ with $V^{u}(y)$ for $y \in B \cap \tilde{\Lambda}_{\varepsilon}$ is transversal.

If $T_{1}$ and $T_{2}$ are two such transversals there is a transition map $\tau$ from a subset of $T_{1}$ to $T_{2}$ defined as follows. If $y \in B \cap \tilde{\Lambda}_{\varepsilon}$ and $V^{u}(y)$ intersects both $T_{1}$ and $T_{2}$, then $\tau\left(T_{1} \cap V^{u}(y)\right)=T_{2} \cap V^{u}(y)$.

If $S$ is a $C^{1}$ submanifold of $M$, let $\lambda_{S}$ denote the measure induced on $S$ by the Riemannian metric of $M$.

THEOREM 2.5. (Pesin) For each $\varepsilon>0$, there is $r(\varepsilon)>0$, with the property that if $x \in \tilde{\Lambda}_{\varepsilon}$ is a density point of $\tilde{\Lambda}_{\varepsilon}, T_{1}$ and $T_{2}$ are $\tilde{\Lambda}_{\varepsilon}$-transversals to $V^{u}(x)$ in $B(x, r(\varepsilon))$ and $\tau$ is the transition map defined above, then $\lambda_{T_{1}}(\operatorname{Dom} \tau)>0, \lambda_{T_{2}}(\operatorname{Im} \tau)>0$ and $\tau$ is absolutely continuous with respect to $\lambda_{T_{1}}$ and $\lambda_{T_{2}}$.

Proof. This follows from the analogue for flows of Theorem 4.4 in [P].

COROllary 2.6. The function $r(\varepsilon)$ in Theorem 2.5 may be chosen so that if $x \in \tilde{\Lambda}_{\varepsilon}$ is a density point of $\tilde{\Lambda}_{\varepsilon}$, the following properties of a measurable set $A \subseteq B(x, r(\varepsilon)) \cap \tilde{\Lambda}_{\varepsilon}$ are equivalent:

(i) $\mu(A)=0$;

(ii) $\lambda_{V^{\prime \prime}(y)}\left(V^{u}(y) \cap A\right)=0$ for $\mu$-a.e. $y \in B(x, r(\varepsilon)) \cap \tilde{\Lambda}_{\varepsilon}$.

Proof. It follows from (iii) of Theorem 2.4 that $r(\varepsilon)$ can be chosen so that for every $x \in \tilde{\Lambda}_{\epsilon}, B(x, r(\varepsilon))$ has a $C^{\prime}$-foliation in which every leaf is a $\tilde{\Lambda}_{\varepsilon}$-transversal to $V^{u}(x)$ in $B(x, r(\varepsilon))$. The corollary follows from Theorem 2.5 and Fubini's theorem.

We shall lise the following global version of Corollary 2.6.

COROllary 2.7. Let $A \subseteq \hat{U}$ be $\mu$-measurable. The following are equivalent:

(i) $\mu(A)=0$; 
(ii) $\lambda_{W^{u}(x)}\left(W^{u}(x) \cap A\right)=0$ for $\mu$-a.e. $x \in \tilde{\Lambda}$;

(iii) $\lambda_{W^{s}(x)}\left(W^{s}(x) \cap A\right)=0$ for $\mu$-a.e. $x \in \tilde{\Lambda}$.

Proof. $(i) \Rightarrow(i i)$. Assume $\mu(A)=0$. Then $\hat{A} \equiv \bigcup_{n \in \mathbb{Z}} \varphi^{n} A$ has $\mu(\hat{A})=0$. Fix $\varepsilon>0$. Let $\hat{A_{\varepsilon}}=\hat{A} \cap \tilde{\Lambda}_{\varepsilon}$. It follows from Corollary 2.6 that for $\mu$-a.e. $y \in \tilde{\Lambda}_{\varepsilon}$,

$$
\lambda_{V^{u}(y)}\left(V_{r(\varepsilon) / 2}^{u}(y) \cap \hat{A}_{\varepsilon}\right)=0,
$$

where $V_{r(\varepsilon) / 2}^{u}(y)$ is the image of $[-r(\varepsilon) / 2, r(\varepsilon) / 2]$ under the map $\alpha^{\mu}(y, \cdot)$ from Theorem 2.3. Let $H_{\varepsilon}=\left\{y \in \tilde{\Lambda}_{\varepsilon}\right.$ : (1) holds $\}$. For $\mu$-a.e. $x \in \tilde{\Lambda}_{\varepsilon}, \varphi^{-n} x \in H_{\varepsilon}$ for arbitrarily large $n \in \mathbb{Z}^{+}$. For such $x$ it follows from Theorem 2.3 that $W^{u}(x)=$ $\bigcup\left\{\varphi^{n} V_{r(\varepsilon) / 2}^{u}\left(\varphi^{-n} x\right): n \geqslant 0\right.$ and $\left.\varphi^{-n} x \in H_{\varepsilon}\right\}$ and consequently $\lambda_{W^{u}(x)}\left(W^{u}(x) \cap \hat{A}_{\varepsilon}\right)=$ 0 . Since $\mu\left(\hat{A} \backslash \bigcup_{n>0} \hat{A}_{1 / n}\right)=0$, it follows that $\lambda_{W^{u}(x)}\left(W^{u}(x) \cap \hat{A}\right)=0$ for $\mu$-a.e. $x \in \tilde{\Lambda}$.

(ii) $\Rightarrow(i)$. This follows easily from Corollary 2.6 .

(i) $\Leftrightarrow($ iii). The proof is analogous to that of (i) $\Leftrightarrow$ (ii).

We need one more consequence of absolute continuity. If $x \in \tilde{\Lambda}$, we shall call $W^{s}(x)$ or $W^{u}(x)$ long if it contains arbitrarily long $C^{1}$ curves with $x$ as midpoint.

Proposition 2.8. Both $W^{s}(x)$ and $W^{u}(x)$ are long for $\mu$-a.e. $x \in \tilde{\Lambda}$.

Proof. We consider only $W^{u}$, since the argument for $W^{s}$ is very similar. If $x \in \tilde{\Lambda}$, let $V^{u+}(x)$ and $V^{u-}(x)$ be the images of $[0, \delta(x)]$ and $[-\delta(x), 0]$ respectively under the map $\alpha^{u}(x, \cdot)$ defined in Theorem 2.3. For $\eta>0$, let

$$
\tilde{\Lambda}_{\varepsilon, \eta}=\left\{x \in \tilde{\Lambda}_{\varepsilon}: \lambda_{V^{u}(x)}\left(V^{u+}(x) \cap \tilde{\Lambda}_{\eta}\right)>\eta \quad \text { and } \quad \lambda_{V^{\prime \prime}(x)}\left(V^{u-}(x) \cap \tilde{\Lambda}_{\eta}\right)>\eta\right\} .
$$

It follows from Theorem 2.3(ii) and Theorem 2.4(iv) that $W^{u}(x)$ is long if $\varphi^{-t} x \in \tilde{\Lambda}_{\varepsilon, \eta}$ for arbitrarily large $t$. The Poincaré recurrence theorem implies that $\mu$-a.e. $x \in \tilde{\Lambda}_{\varepsilon, \eta}$ has $\varphi^{-t} x \in \tilde{\Lambda}_{\varepsilon, \eta}$ for arbitrarily large $t$. Since $\bigcup_{\varepsilon>0} \tilde{\Lambda}_{\varepsilon}$ has full measure in $\tilde{\Lambda}$, the proposition follows from Lemma 2.9 below.

LEMMA 2.9. For each $\varepsilon>0$,

$$
\mu\left(\tilde{\Lambda}_{\varepsilon} \backslash \tilde{\Lambda}_{\varepsilon, \eta}\right) \rightarrow 0 \quad \text { as } \eta \rightarrow 0 .
$$

Proof. If not, there is a $\mu$-measurable set $B \subseteq \tilde{\Lambda}_{\varepsilon}$ such that $\mu(B)>0$ and $B \cap \tilde{\Lambda}_{\varepsilon, \eta}=\varnothing$ for every $\eta>0$. It follows from Corollary 2.6 that there is $y \in \tilde{\Lambda}_{\varepsilon}$ such that $\lambda_{V^{u}(y)}\left(V^{u}(y) \cap B\right)>0$. Clearly any $b \in V^{u}(y) \cap B$ which is a density point (w.r.t. $\left.\lambda_{V^{u}(y)}\right)$ of $V^{u}(y) \cap B$ is in $\tilde{\Lambda}_{\varepsilon, \eta}$ for some $\eta>0$, contradicting the definition of $B$.

\section{Ergodicity on $\hat{U}$}

Hypothesis (iv) of Theorem 1.1 and the continuity of the cone family $\mathscr{K}(x)$ imply that each $x \in U$ has a neighborhood $U_{x} \subseteq U$ with

$$
d \varphi^{-t(x)} \mathscr{K}^{*}\left(\varphi^{t(x)} y\right) \subseteq \operatorname{Int} \mathscr{K}^{*}(y)
$$

for each $y \in U_{x}$. It follows easily that there is a continuous family of cones $\mathscr{K}^{-}(x)$ in $P$ over $U$ such that, for each $x \in U$,

$$
\begin{gathered}
\mathscr{K}^{-}(x) \subseteq \operatorname{Int}\left(\mathscr{K}^{*}(x)\right), \\
\bigcap\left\{d \varphi^{-t} \mathscr{K}^{*}\left(\varphi^{t} x\right): t \geq 0 \text { and } \varphi^{\prime} x \in U\right\} \subseteq \operatorname{Int} \mathscr{K}^{-}(x) .
\end{gathered}
$$


It follows from (3), Theorem 2.2(iv) and the invariance properties of $\mathscr{K}$ and $E^{s}$ that

$$
E^{s}(x) \subset \mathscr{K}^{-}(x) \text { if } x \in \Lambda \cap U .
$$

LEMMA 3.1. For $\mu$-a.e. $x \in \tilde{\Lambda}$, we have $T_{y} W^{u}(x) \subset \mathscr{K}(y)$ for every $y \in W^{u}(x) \cap U$ and $T_{z} W^{s}(x) \subset \mathscr{K}^{-}(z)$ for every $z \in W^{s}(x) \cap U$.

Proof. If $y \in W^{u}(x) \cap U \cap \tilde{\Lambda}$, then $T_{y} W^{u}(x)=E^{u}(y) \subseteq \mathscr{K}(y)$ by Theorem 2.3(v) and (iii), and Theorem 2.2(iv). Similarly if $z \in W^{s}(x) \cap U \cap \tilde{\Lambda}$, then $T_{y} W^{s}(x)=E^{s}(y) \subseteq$ $\mathscr{K}^{-}(y)$ by (3.4). By Corollary 2.6, $\mu$-a.e. $x \in \tilde{\Lambda}$ has

$$
\lambda_{W^{s}(x)}\left(W^{s}(x) \backslash \tilde{\Lambda}\right)=0=\lambda_{W^{u}(x)}\left(W^{u}(x) \backslash \tilde{\Lambda}\right) .
$$

The lemma now follows since $W^{s}(x)$ and $W^{u}(x)$ are $C^{1}$-immersed submanifolds and the cone families $\mathscr{K}$ and $\mathscr{K}^{-}$are continuous.

Let $\Lambda^{*}$ be the set of points in $\tilde{\Lambda} \cap U$ for which (5) and the conclusions of Proposition 2.8 and Lemma 3.1 hold. Observe that

$$
\mu\left(U \backslash \Lambda^{*}\right)=0 .
$$

Call a $C^{1}$ curve $\sigma$ in $U$ a $\mathscr{K}$-curve or a $\mathscr{K}^{-}$-curve if $T_{x} \sigma \in \mathscr{K}(x)$ or $\mathscr{K}^{-}(x)$ respectively for each $x \in \sigma$. A surface in $U$ of the form $\bigcup\left\{\varphi^{t} \sigma:|t|<T\right\}$, where $\sigma$ is a $\mathscr{K}^{-}$-curve, will be called a $\mathscr{K}^{-}$-surface. Since the cone families $\mathscr{K}$ and $\mathscr{K}^{-}$are continuous on $U$ and $\mathscr{K}^{-} \subseteq \operatorname{Int}\left(\mathscr{K}^{*}\right)$, it is clear that for each $x \in U$ there are $\rho(x)>0$ and neighborhoods $N_{1}(x)$ and $N_{2}(x)$ of $x$ such that $N_{1}(x) \subseteq N_{2}(x) \subseteq U$ and the following properties hold:

(i) Any intersection in $N_{2}(x)$ of a $\mathscr{K}$-curve and a $\mathscr{K}^{-}$-surface is transversal.

(ii) Suppose $\sigma_{1}$ and $\sigma_{2}$ are $\mathscr{K}$ and $\mathscr{K}^{-}$curves respectively, each with length at least

$2 \rho(x)$ and midpoint in $N_{1}(x)$. Then $\sigma_{1}(x)$ and the $\mathscr{K}^{-}$-surface $\bigcup\left\{\varphi^{\prime} \sigma_{2}:|t| \leq\right.$ $\rho(x)$ \} intersect in $N_{2}(x)$.

It follows from Lemma 3.1 and Proposition 2.8 that, if $y \in \Lambda^{*}$ then each component of $W^{u}(y) \cap U$ (in the submanifold topology on $W^{u}(y)$ ) is a $\mathscr{K}$-curve and each component of $W^{s}(y) \cap U$ is a $\mathscr{K}^{-}$-curve.

Proof of Theorem 1.1. For a continuous function $f: M \rightarrow \mathbb{R}$, set

$$
\hat{f}^{+}(x)=\lim _{T \rightarrow+\infty} \frac{1}{T} \int_{0}^{T} f\left(\varphi^{t} x\right) d t \text { and } \hat{f}^{-}(x)=\lim _{T \rightarrow+\infty} \frac{1}{T} \int_{0}^{T} f\left(\varphi^{-t} x\right) d t .
$$

Ergodicity of $\varphi^{t}$ on $\hat{U}$ will follow if we prove that $\hat{f}^{+}$and $\hat{f}^{-}$are constant on a subset of $U$ with full measure. We shall show that for every $x \in U$ the neighborhood $N_{1}(x)$ defined above has a subset of full measure on which $\hat{f}^{+}$and $\hat{f}^{-}$are constant. Let $G=\left\{x \in \Lambda^{*}: \hat{f}^{+}(x)\right.$ and $\hat{f}^{-}(x)$ are defined and equal $\}$. It follows from (6) and the Birkhoff ergodic theorem that $\mu(U \backslash G)=0$. By Corollary 2.7, there is $y \in N_{1}(x) \cap$ $G$ such that $\lambda_{W^{s}(y)}\left(W^{s}(y) \backslash G\right)=0$. Since the set $G$ is $\varphi^{t}$-invariant, we have

$$
\lambda_{W^{0 s}(y)}\left(W^{0 s}(y) \backslash G\right)=0 .
$$

By Lemma 3.2 below, for $\mu$-a.e. $z \in G \cap N_{1}(x)$ there is a point $p \in W^{u}(z) \cap W^{0 s}(y) \cap$ $N_{2}(x) \cap G$. Since $z \in G$ and $z$ and $p$ lie in the same unstable manifold, $\hat{f}^{+}(z)=$ $\hat{f}^{-}(z)=\hat{f}^{-}(p)$. Also, $\hat{f}^{-}(p)=\hat{f}^{+}(p)$ because $p \in G$. Since $p$ and $y$ lie in the same weak stable manifold, $\hat{f}^{+}(p)=\hat{f}^{+}(y)$. Thus $\hat{f}^{+}(z)=\hat{f}^{-}(z)=\hat{f}^{+}(y)$ for $\mu$-a.e. $z \in$ $N_{1}(x)$. 
LemMA 3.2. Let $x \in U$ and $y \in \Lambda^{*} \cap N_{1}(x)$. Set

$$
\begin{aligned}
A=\bigcup\left\{W^{u}(z):\right. & z \in \Lambda^{*} \cap N_{1}(x) \\
& \text { and } \left.W^{u}(z) \cap W^{0 s}(y) \cap N_{2}(x) \cap G=\varnothing\right\} .
\end{aligned}
$$

Then $\mu(A)=0$.

Proof. Suppose $\mu(A)>0$. Then there are $\varepsilon>0$ and $z \in A$ such that $z$ is a density point of $A \cap \tilde{\Lambda}_{\varepsilon}$. We may assume that $\varphi^{-t} z \in \tilde{\Lambda}_{\varepsilon}$ and $\varphi^{-t} z$ is a density point of $\tilde{\Lambda}_{\varepsilon}$ for arbitrarily large positive $t$. Let $z^{\prime} \in W^{u}(z) \cap W^{0 s}(y) \cap\left(N_{2}(x) \backslash G\right)$ and let $\beta$ be the piece of $W^{u}(z)$ that joins $z$ to $z^{\prime}$. Choose $t_{0}>0$ such that $\varphi^{-t_{0}} z \in \tilde{\Lambda}_{\varepsilon}$ is a density point of $\tilde{\Lambda}_{\varepsilon}$ and $\varphi^{-t_{0}} \beta$ lies in the ball $B\left(\varphi^{-t_{0}} z, r(\varepsilon)\right)$, where $r(\varepsilon)$ is defined by Theorem 2.5. Observe that $\varphi^{-t_{0}} z$ is a density point of $\tilde{\Lambda}_{\varepsilon} \cap \varphi^{-t_{0}} A$ and $\varphi^{-t_{0}} A$ is a union of global unstable manifolds. It is clear from Theorem 2.5 that any neighborhood $Q$ of $\varphi^{-t_{0} z}$ in $W^{0 s}\left(\varphi^{-t_{0}} z\right)$ has $\lambda_{W^{0 s}\left(\varphi^{-t_{0} z}\right)}\left(Q \cap \varphi^{-t_{0}} A\right)>0$. It follows from Theorem 2.5 that any submanifold $T$ of $M$ that intersects $W^{u}\left(\varphi^{-t_{0}} z\right)$ transversally at a point $z^{\prime \prime} \in B\left(\varphi^{-t_{0}} z, r(\varepsilon)\right)$ has $\lambda_{T}\left(Q^{\prime} \cap \varphi^{-t_{0}} A\right)>0$ for every neighborhood $Q^{\prime}$ of $z^{\prime \prime}$ in $T$. Since $W^{0 s}(y)$ and $W^{u}(z)$ intersect transversally at $z^{\prime}$, we may take $T=$ $\varphi^{-t_{0}} W^{0 s}(y)$ and $z^{\prime \prime}=\varphi^{-t_{0}} z^{\prime}$. It follows that $\lambda_{W^{0 s}(y)}\left(W^{0 s}(y) \cap N_{2}(x) \cap A\right)>0$. But this contradicts (7), since $W^{0 s}(y) \cap N_{2}(x) \cap A \cap G=\varnothing$.

\section{REFERENCES}

[BG1] K. Burns \& M. Gerber. Bernoulli geodesic flows on $S^{2}$. Preprint, 1987.

[BG2] K. Burns \& M. Gerber. Real analytic Bernoulli geodesic flows on $S^{2}$. Preprint, 1987. To appear in Ergod. Th. \& Dynam. Sys.

[D1] V. J. Donnay. Geodesic flow on $S^{2}$ with positive measure entropy. Preprint, 1986.

[D2] V. J. Donnay. Geodesic flow on the two-sphere with positive entropy. Ph.D. Thesis, New York University, 1986.

[D3] V. J. Donnay. Geodesic fiow on the two-sphere, Part I: Positive measure entropy. Preprint, 1987.

[D4] V. J. Donnay. Geodesic flow on the two-sphere, Part II: Ergodicity. Preprint, 1987.

[FHY] A. Fathi, M. R. Herman, J. C. Yoccoz. A proof of Pesin's stable manifold theorem. Lecture Notes in Mathematics 1007, pp. 177-215. Springer: New York, 1983.

[K] A. Katok. Invariant cone families and stochastic properties of smooth dynamical systems. Preprint, 1987.

[O] V. I. Osceledec. A multiplicative ergodic theorem: characteristic Lyapunov exponents of dynamical systems. Trans. Moscow Math. Soc. 19 (1968), 197-231.

[P] Ya. B. Pesin. Characteristic Lyapunov exponents and smooth ergodic theory. Russian Math. Surveys 32 (1977), 55-114.

[W] M. Wojtkowski. Invariant families of cones and Lyapunov exponents. Ergod. Th. \& Dynam. Sys. 5 (1985), 145-161. 